Endzeiten

\begin{tabular}{l} 
W \\
DE \\
\hline
\end{tabular} 


\title{
Millennium-Studien
}

zu Kultur und Geschichte des ersten Jahrtausends n. Chr.

\section{Millennium Studies}

in the culture and history of the first millennium C.E.

\author{
Herausgegeben von / Edited by \\ Wolfram Brandes, Alexander Demandt, Helmut Krasser, \\ Hartmut Leppin, Peter von Möllendorff
}

Band 16

Walter de Gruyter · Berlin · New York 


\title{
Endzeiten
}

\author{
Eschatologie in den \\ monotheistischen Weltreligionen
}

herausgegeben von

Wolfram Brandes und Felicitas Schmieder

Walter de Gruyter · Berlin · New York 
Diese Publikation wurde im Rahmen des Fördervorhabens 16TOA021 - Reihentransformation für die Altertumswissenschaften („Millennium-Studien“) mit Mitteln des Bundesministeriums für Bildung und Forschung im

Open Access bereitgestellt. Das Fördervorhaben wird in Kooperation mit dem DFG-geförderten Fachinformationsdienst Altertumswissenschaften - Propylaeum an der Bayerischen Staatsbibliothek durchgeführt.

\section{(cc) BY-NC-ND}

Dieses Werk ist lizenziert unter der Creative Commons Attribution-NonCommercial-NoDerivatives 4.0 International Lizenz. Weitere Informationen finden Sie unter http://creativecommons.org/licenses/by-nc-nd/4.0/.

Die Bedingungen der Creative-Commons-Lizenz gelten nur für Originalmaterial. Die Wiederverwenung von Material aus anderen Quellen (gekennzeichnet mit Quellenangabe) wie z.B. Schaubilder, Abbildungen, Fotos und Textauszüge erfordert ggf. weitere Nutzungsgenehmigungen durch den jeweiligen Rechteinhaber.

@ Gedruckt auf säurefreiem Papier, das die US-ANSI-Norm über Haltbarkeit erfüllt.

ISBN 978-3-11-018621-5

ISSN 1862-1139

Bibliografische Information der Deutschen Nationalbibliothek

Die Deutsche Nationalbibliothek verzeichnet diese Publikation in der Deutschen Nationalbibliografie; detaillierte bibliografische Daten sind im Internet über http://dnb.d-nb.de abrufbar.

(C) Copyright 2008 by Walter de Gruyter GmbH \& Co. KG, 10785 Berlin

Dieses Werk einschließlich aller seiner Teile ist urheberrechtlich geschützt. Jede Verwertung außerhalb der engen Grenzen des Urheberrechtsgesetzes ist ohne Zustimmung des Verlages unzulässig und strafbar. Das gilt insbesondere für Vervielfältigungen, Übersetzungen, Mikroverfilmungen und die Einspeicherung und Verarbeitung in elektronischen Systemen.

Printed in Germany

Umschlaggestaltung: Christopher Schneider, Berlin 


\section{Einleitung}

Vom 31. 3. bis zum 2. 4. 2005 fand in Frankfurt die Tagung statt, deren Vorträge im vorliegenden Band publiziert werden. Wir danken insbesondere der Fritz-Thyssen-Stiftung, deren großzügige und unbürokratische Unterstützung eine wesentliche Voraussetzung für die erfolgreiche Durchführung war.

Die drei genetisch eng miteinander verbundenen monotheistischen Weltreligionen (Judentum, Christentum und Islam) kennen vergleichbare Vorstellungen vom Ende der Zeiten. Von gemeinsamen Konzepten ausgehend differierten und konkurrierten diese im Laufe der Zeit bis heute, und zwar innerhalb der einzelnen Religionsgemeinschaften ebenso wie im Zusammenspiel mit den anderen. Denn die drei traditionell miteinander verschränkten Großgruppen greifen in hohem Maße auf die gleichen Texte zurück, seien sie nun "heilig“ oder „legendär" (wie der „Alexander-Roman“). Nicht zuletzt weisen sich diese Gemeinschaften gegenseitig stets eschatologische Rollen in der jeweils eigenen Sicht zu.

Dabei ist zu betonen, was in der modernen Forschung vielfach allzu gerne unterschätzt wird: Prophetie wurde verstanden - ihre Bilder waren den Zeitgenossen, sofern sie eine gewisse Bildung besaßen, vertraut und nicht zuletzt die selbstverständliche Überzeugung, daß Gott die Welt zu einem bestimmten Zeitpunkt geschaffen habe und sie auch zu einem vorherbestimmten Zeitpunkt wieder vernichten würde. Dieses Wissen konnte nicht nur als Hoffnungs- und Drohkulisse dienen, sondern es bildete den Rahmen für Denken und Handeln der Zeitgenossen. Deshalb entfalteten Deutungen alter Prophetien und neugeschaffene Vorhersagen soziale und politische Wirksamkeit; ja, sie konnten im politischen Spiel benutzt und manipuliert werden. Doch schließt die pragmatische Manipulation eines Textes und der Einsatz bestimmter Medien zu dessen Verbreitung nicht seine grundsätzliche Wirkmächtigkeit aus. Wir haben davon auszugehen, daß den Zeitgenossen der Kontext von Prophezeiungen klar war das heißt aber nicht, daß sie und auch die Verfertiger dieser politischen Warnungen das Szenario als solches für phantastisch gehalten hätten. Denn die Überzeugung vom göttlichen Wirken in der Welt, vom Anfang und dem sicheren Ende der Geschichte, von einer Heilsgeschichte, deren Ablauf vorbestimmt und biblisch versprochen war, stand nicht zur Disposition, war nicht Gegenstand der Manipulation, sondern ihr Rahmen, ihr funktionaler Hintergrund, ihr Nährboden, ihre Voraussetzungen, aus denen alle Beteiligten ihr Wissen und ihre Ideen zogen. 
Man vergriff sich nicht am Namen Gottes, man spielte nicht ungestraft mit der göttlichen Sehergabe. Deshalb waren selbst die Autoren der Prophezeiungen davon überzeugt, daß sie die Wahrheit sprachen - aber diese Wahrheit in ein für die, die hören sollten und es vielleicht nicht wollten, überzeugendes Gewand kleideten, so daß sie nicht einfach übergangen werden konnte. Letztlich ist der überzeugendste „Demagoge“ der, der fest an seine eigene Sendung glaubt, wie sehr er sich auch bewußt ist, daß er Tatsachen beugt, um die Wahrheit zu vermitteln. Kurz, ein solcher symbolischer Diskurs funktioniert nur, wenn alle Beteiligten an seine Wirkmacht glauben und seine Grundlagen teilen.

In diesem Sinne war Prophetie immer politisch und hatte eine besondere Position in der tagespolitischen Diskussion. Das gilt für die nicht-christliche Antike ebenso wie für die altorientalischen und auch ganz andere Kulturen, soweit wir sie gut genug kennen - und es gilt für das Judentum, für das Christentum seit der Spätantike und ebenso für den Islam. Christliche Prophetie beschäftigt sich mit der gesamten Heilsgeschichte seit ihren Anfängen und bis zu ihrem Ende, ist deshalb die Gattung, in der man Deutungsmodelle der Weltgeschichte findet. Und immer wieder ist diese Geschichtsdeutung zugespitzt auf die Zeitgeschichte, positioniert sie die Jetztzeit in der Heilsgeschichte, ist sie politische Zeitdeutung - die wiederum für sich allein stehen konnte, dabei aber immer implizit auf den heilsgeschichtlichen Gesamtkontext verwies.

Die wichtigste Qualiät der Prophetie bestand stets in der richtigen Interpretation der „Zeichen“ der Zeit: Je mehr Zeichen erkannt und gelesen wurden, desto wertvoller war eine Prophezeiung und ihre Deutung. Um geglaubt zu werden, mußte eine Prophezeiung hochaktuell sein, mußte sie extreme Flexibilität zeigen und lebendiges Denken repräsentieren. Sie mußte Fragen beantworten. Und da die Prophezeiung die zentrale Frage nach der Zukunft christlicher Existenz stellte und die Tagesereignisse einschloß, reagierte sie zugleich auf diese Ereignisse. So konnte sie ganz bewußt als Medium politischer Propaganda eingesetzt werden - sie konnte mit hoher Autorität Handlungsanweisungen zu geben versuchen. Denn während jede andere Propaganda Lösungen nur versprechen konnte, konnte die Prophetie - die sich per definitionem mit der Zukunft beschäftigt - Probleme lösen!

Dieser permanente Prozeß der symbolischen Kommunikation zwischen Vergangenheit und Gegenwart über eine für erschließbar gehaltene Zukunft hat zentrale Bedeutung für das religiöse, kulturelle und nicht zuletzt politische Leben. Bestimmte Ereignisse werden in realen (z. B. das irdische Jerusalem) oder/und imaginären (z. B. das irdische Paradies, das himmlische Jerusalem) Räumen verortet. Von den Kontaktzonen der monotheistischen Weltreligionen (insbes. Syrien/Palästina und Süditalien) aus werden Ideen entwickelt und weiter verbreitet, auch über die Grenzen der Kulturräume hinweg.

Erwartet wird stets, daß am Ende der Zeiten eine Rettergestalt (Messias, Endzeitkaiser, Mahdi) noch vor dem Letzten Gericht auftreten wird, als Weg- 
weiser in ein Reich des Friedens und des endgültigen Triumphs der Gläubigen. Um den Zeitpunkt als nah zu erkennen, werden bestimmte Zeichen, die in alten (oft in Heiligen Schriften, wie dem Alten und Neuen Testament sowie dem Koran) oder neu inspirierten Prophezeiungen niedergelegt sind, als Übereinstimmungen mit Zeitereignissen gedeutet. Dabei kann die Stellung und Akzeptanz aktueller Propheten in den verschiedenen Religionsgemeinschaften und über die Zeiten sehr unterschiedlich sein: Wenn etwa im Islam die Möglichkeit von Prophetie nach Mohammed, dem "Siegel der Propheten“, als heterodox abgestempelt ist und subsidiäre Erscheinungen etwa in der Mystik leicht verdächtig erscheinen - oder wenn im lateinischen Christentum des Spätmittelalters die unruhigen Folgen begeistert aufgenommener spiritueller Prophetie die wissenschaftlich-definitorische Suche nach falsi prophetae nach sich ziehen.

Immer wieder aber lassen in allen betrachteten Religionen aktuelle Katastrophen (Erdbeben, Seuchen, 11. 9., astronomische und meteorologische Phänomene usw.) sowie "gefährliche“ Wendezeiten, die sich aus den jeweiligen Zeitberechungssystemen ergeben, Endzeiterwartungen besonders virulent werden. Allein schon die verschiedenen Systeme der christlichen Weltalterberechnungen führten um die Jahre 500, 800 und 1000 a. D. zu Erwartungen, daß das Ende der Welt unmittelbar bevorstehen könnte. Ähnliches läßt sich im Islam (Mahdi-Erwartungen) und im Judentum (Messias-Erwartung zum Jahr $5000=$ 1240/ 41 - falls es sie denn gegeben hat) beobachten.

Dies hatte massive Auswirkungen auf die jeweils aktuellen Vorstellungen von Zeit, Welt, Religion, Kunst oder Gesellschaft insgesamt - und da es sich normalerweise um Herrschaftswissen und seinen gezielten Einsatz handelte, war fast immer auch die "große“ Politik zutiefst involviert. Das Auftreten von Propheten oder eschatologischen Zeichendeutern ist fast stets im Kontext konkreter politischer Propaganda - von Versuchen, bestimmte Aktionen zu initiieren oder zu verhindern - zu sehen.

Diese Tatsache wird gerade in der europäischen Forschung viel zu wenig beachtet, oft genug bewußt ignoriert. So erschien anläßlich des Millenniums 2000 eine kaum übersehbare Flut von Publikationen unterschiedlichsten Niveaus (mit verschiedensten Adressaten), die leider viel zu oft eine ausgewogene wissenschaftliche Behandlung der Endzeitproblematik vermissen ließ. Auch die wissenschaftliche Diskussion in der Mediävistik (besonders in Deutschland und Frankreich) zum Problem des Jahres 1000 litt unter dem erstaunlichen Manko, welches von mediävistischer Seite kaum bemerkt, geschweige denn thematisiert wurde: Der christliche Osten, das Byzantinische Reich und der sog. Oriens christianus (die verschiedenen christlichen Kirchen im islamischen Herrschaftsbereich) wurden weitgehend ignoriert. Wie im Westen gab es in Byzanz eine auf die Jahre um 1000 a. D. gerichtete Endzeiterwartung (auf ganz eigener chronologischer Grundlage), die zudem nachweisbar mit der im lateinischen Westen in Wechselwirkung stand. 
Für diesen Band und die ihm zugrundeliegende Tagung haben wir uns deshalb bemüht, das Problem der christlichen Endzeiterwartung der ersten anderthalb Jahrtausende mit dem nötigen Abstand historisch ernsthaft sowie transdisziplinär und unter Berücksichtigung verschiedener mediterraner Kulturräume zu behandeln. Vergleichend, aber auch in ihrem Eigenwert, sind die jüdischen und muslimischen Konzepte herangezogen worden, ohne die auch die christlichen Vorstellungen nicht zu verstehen sind. Dabei galt es nicht zuletzt, die Begrifflichkeit der bisherigen Debatten neu zu ordnen. Schon eine kursorische Durchsicht etwa der zahlreichen Publikationen pro oder contra eine Endzeiterwartung um das Jahr 1000 läßt einen erstaunlich laxen Umgang mit den (oft nur scheinbar) diskutierten Begriffen („Endzeit“, „Endzeiterwartung“„Eschatologie“, „Apokalyptik“, „Prophetie“ - „Millennium“, „Chiliasmus“ usw.) erkennen. Da jeweilige historische Endzeiterwartungen bzw. Endzeitreferenzen in der Literatur oder Kunst stets durch ganz spezifische geschichtliche Kontexte bedingt waren, muß bei der Analyse der herangezogenen Zeugnisse z. B. von einer strikten Beachtung ihrer Historizität ausgegangen werden - eine Binsenweisheit, die leider zu oft ignoriert wird.

Der besondere Fokus ist der zeitpolitischen intendierten wie tatsächlichen Wirksamkeit zugedacht, aufgrund der Erkenntnisse der Forschung der letzten Jahre, daß sich prophetisch-apokalyptische Schriften sehr oft im Zentrum der Mächtigen dieser Welt bewegten und die zentralen Fragen der Zeit aufnahmen und wirkungsvoll kommentierten. Derartiges Schriftgut ist dementsprechend als wichtige Quelle nicht nur der Geistes- sondern auch der politischen Geschichte ernst zu nehmen. Gerade die Betonung dieses Aspekts stellte einen der Schwerpunkte mehrerer Vorträge und besonders der Diskussionen dar.

Die Beiträge des vorliegenden Bandes repräsentieren gemäß seinem transdisziplinären Anliegen folgende wissenschaftliche Disziplinen: Oriens Christianus (syrische, armenische usw. Kirchen), spätantike Religionsgeschichte und Byzanz, die lateinische Kirche des Mittelalters und die europäischen Reformationsbewegungen, sodann das Judentum und den Islam (Schiiten wie Sunniten). Mit dem interdisziplinären Zugang wurden auf der Tagung direkt unterschiedliche Forschungstraditionen und Quellensprachen miteinander ins Gespräch gebracht und vernetzt. Dies kann in vielen Bereichen die bisherige Forschung weitgehend auf den Kopf stellen, weil plötzlich Begründungen und Parallelbeispiele zur Verfügung gestellt werden, die nicht oder kaum wahrgenommen wurden.

So sehr sich die geplante Tagung mit vergangenen Denk- und Handlungsweisen beschäftigen soll, hat sie doch eminent bedeutsame Gegenwartsbezüge. Endzeitlich geprägte politische Rhetorik („Achse des Bösen“, „Großer Satan“) beziehen sich auf zentrale Motivationsmuster, die hinter den als „Clash of Civilizations“ oder auch „Krieg gegen den Terror“ apostrophierten, weltbewegenden politisch-kulturellen Konflikten stehen. Fundamentalistisch musli- 
misch, jüdisch und christlich geprägte politische Überzeugungen finden sich auf allen Seiten, und jede von ihnen sieht sich selbst in einer Gegenwartsgeschichte, die auf ein (meist unmittelbar) bevorstehendes, siegreich zu bewältigendes Ende der Geschichte zuläuft. Allerdings liegt hochsäkularisierten Gesellschaften diese Art religiös motivierten Planens und Handelns fern und ist ihrer politischen Kultur so fremd geworden, daß sie sie gar nicht erkennt, nicht als wirkmächtig ernst nimmt, oder aber ihre Wirkmächtigkeit nicht einschätzen kann.

Deshalb hat sich an erster Stelle Andrew Colin Gow („Wie übergeht oder ignoriert man eschatologisches Gedankengut? Und warum? Und wenn man es einmal übergangen oder verkannt hat, was wären dann konkret die Folgen?") dieser Tatsache angenommen und die Existenz sowie die Gefahren moderner gesellschaftlicher und politischer eschatologischer Kräfte US-amerikanischer Prägung aufgezeigt. Zuvor aber wendet er sich den historischen Endzeitbildern zu (die in der Gegenwart mutatis mutandis weiterleben und deren Kenntnis schon allein zu deren Verständnis notwendig ist) und beleuchtet das in der modernen Forschung zur lateinisch-christlichen Geschichte (und nicht nur da) verbreitete Zögern im Umgang mit eschatologischem Gedankengut und den dieses transportierenden Quellen, denen häufig jegliche zeitgenössische Relevanz abgesprochen wurde und wird. Gow bringt diese Erscheinung (neben säkularen Tendenzen) vorrangig mit der Tatsache in Verbindung, daß das mittelalterliche Antichristbild stark antijüdische Züge trug.

Den radikalarianischen (eunomianischen) Kirchenhistoriker Philostorgios stellt Bruno Bleckmann („Apokalypse und kosmische Katastrophen: Das Bild der theodosianischen Dynastie beim Kirchenhistoriker Philostorg") ins Zentrum seiner Überlegungen und weist vor allem auf den Einsatz endzeitlicher Topoi und Formeln zur dynastischen Kritik hin. Die in der Forschung verbreitete Annahme, daß Philostorgios direkt auf der Apokalypse des sog. Testamentum Domini basiere, lehnt er mit guten Gründen ab. Statt dessen betont er die Existenz eschatologischer Vorstellungen z. Z. Theodosios' I., die eben auch bei Philostorgios einen Niederschlag fanden.

Mischa Meier („Eschatologie und Kommunikation im 6. Jahrhundert n. Chr. - oder: Wie Osten und Westen beständig aneinander vorbei redeten") versteht seinen Beitrag im Rahmen der Frage nach der Transformation des Imperium Romanum in ein Europa und greift anhand des Themas eine viel umfassendere Fragestellung auf: Dabei wendet er sich gegen manches historiographisches Konstrukt von „Kommunikation“ und plädiert dafür, daß über den bloßen Kontakt hinausgehendes Verstehen vonnöten sei. Zur Überprüfung tauge die Eschatologie als Thema, das beide Seiten interessiere; Beispiele um 400, um 500 und um 600 werden aufgegriffen. Und hier zeigt sich eine deutliche Verringerung (entgegen der vielleicht doch etwas zu optimistischen Ansicht von Michael McCormick): Gab es bis um Beginn des 6. Jhs. noch einen regen Austausch endzeitlicher Vorstellungen und Deutungsmuster der Gegenwart, so gingen 
lateinischer Westen und griechischer Osten spätestens seit der Mitte dieses Jahrhunderts getrennte Wege. Meier deutete dies als einen Abbruch der Kommunikation im Bereich eines derart spezialisierten Wissens.

Hatte schon Meier für verstärkte eschatologische Äußerungen wichtige krisenhafte Zeiten betrachtet, so kann Gerrit J. Reinink („From Apocalyptics to Apologetics: Early Syriac Reactions to Islam“) dieses Problem anhand der für das syrische Christentum existenziellen Bedrohung durch den Islam aufgreifen. Aus Angst vor Massenkonversionen entstandene in vielem ganz unterschiedliche syrische polemisch-apologetische und eschatologische Äußerungen über die muslimischen Araber aus den 690er Jahren zeigen unterschiedliches Wissen über den anderen Glauben und zeichnen die Araber entweder als Leugner und Abgefallene vom rechten Glauben oder gar Mohammed als Kryptochristen. Auch die erwarteten Konsequenzen unterscheiden sich in ihrer Aussicht entweder auf einen gewaltsamen christlichen Endsieg im Diesseits oder den Verweis auf ein himmlisches Reich.

Andrea B. Schmidt („Die ,Brüste des Nordens‘ und Alexanders Mauer gegen Gog und Magog") beschäftigt sich damit, wie sich die verschiedenen orientalisch-christlichen Literaturen mit den vor allem durch die Apokalypse des Pseudo-Methodius bekanntgewordenen von Alexander dem Großen eingeschlossenen apokalyptischen Völker Gog und Magog auseinandersetzten. Dabei geht sie vom originellen syrischen Milieu aus und untersucht die Lokalisierungen der „Mauer des Nordens“: Sie werden stets, als Sicherung gegen bedrohliche Nomadeneinfälle, an unterschiedlichen Stellen im Kaukasus gewußt und wohl deshalb fehlen sie bei Armeniern und Georgiern, denen nicht nur der Kaukasus zu vertraut war, sondern die offenbar auch eine andere Einstellung den nahen Nordvölkern gegenüber entwickelten.

Die Idee der vier Weltreiche aus dem alttestamentlichen Buch Daniel verfolgt Wout J. van Beckum („Four Kingdoms Will Rule: Echoes of Apocalypticism and Political Reality in Late Antiquity and Medieval Judaism") in der frühen rabbinischen Literatur. Eine Vielzahl von Interpretationen der Basiszahl vier und des Grundmusters der Fremdherrschaft als Prüfung Gottes für Israel lassen sich zeigen. Dominant erscheint die jeweilige politische Realität, so die Verschlechterung der Lage der Juden mit dem Aufstieg des Christentums. In knapper Folge ändern sich dementsprechend zum Beginn des 7. Jahrhunderts die Hoffnungen und Befürchtungen, wenn man vor 614 auf die Perser hoffte (infolge von deren Eingreifen bis 617 Jerusalem praktisch jüdisch war), 630 bis 636/7 unter byzantinischen Verfolgungen zu leiden hatte und unter dem Eindruck der muslimischen Siege die Identifikation der vier Reiche maßgeblich uminterpretierte.

In einem Block mehrerer Beiträge zu byzantinischen Endzeitvorstellungen gibt Paul Magdalino („The End of Time in Byzantium“) einen umfassenden und charakterisierenden Überblick über die Entwicklungen eschatologischer 
Schriften und des entsprechenden Gedankengutes in Byzanz durch die Jahrhunderte hindurch.

Albrecht Berger („Das apokalyptische Konstantinopel. Topographisches in apokalyptischen Schriften der mittelbyzantinischen Zeit") zeigt, wie bedeutend (und bisher kaum beachtet) der Einfluß apokalyptischer Vorstellungen von Konstantinopel und dem Ende der Stadt, gleichbedeutend mit dem Ende des Reiches und der Welt auch für die konkreten Vorstellungen von der "Stadt“, bis hin zur konkreten Topographie, war. Er stellt diverse Texte vor, die zu unterschiedlichen Zeiten und in unterschiedlicher Weise die endzeitliche Eroberung und Untergang Konstantinopels thematisieren.

In Byzanz bleibt auch Wolfram Brandes („Kaiserprophetien und Hochverrat. Apokalyptische Schriften und Kaiservatizinien als Medium antikaiserlicher Propaganda"). Er wendet sich mit der Behandlung von drei ausgewählten Beispielen aus verschiedenen Jahrhunderten (als pars pro toto) der Problematik der Verwendung und auch des „Mißbrauchs" apokalyptischer Literaturformen („Kurzapokalypsen“ und einige mit diesen verwandte sog. Kaiserprophetien) in politischen Auseinandersetzungen $\mathrm{zu}$, bis hin $\mathrm{zu}$ einem neu interpretierten Gesetz (von 1280), das das Verfassen solcher Schriften mit dem Tode bedrohte. Besondere Berücksichtigung fand die lateinische Übersetzung solcher Schriften durch Venezianer im 13. Jh.

Dem lateinisch-christlichen Bereich zwischen dem 12. und 15. Jahrhundert wenden sich die folgenden Beiträge zu. Hannes Möhring („Die Weissagungen über einen Kaiser Friedrich am Ende der Zeiten") verfolgt das Motiv eines endzeitlichen Kaisers namens Friedrich in zweifacher Gestalt: Zum einen die Hoffnung auf eine Wiederkehr Friedrichs II., der der Sibylle zufolge „lebt und nicht lebt" (einhergehend mit dem Auftreten diverser „falscher Friedriche"), zum anderen die dies nach einigen Jahrzehnten weitgehend ablösende Erwartung eines Dritten Friedrich. Im Übergangsbereich zwischen beiden Ideenkomplexen liegt die Verbindung der Figur des „Friedrich“ mit dem Endkaiser im 14. Jahrhundert.

Das Machtzentrum der römische Kurie greift Christian Jostmann („Prophetie an der Kurie des 13. Jahrhunderts") auf, der neben der "Sibilla Erithea" drei weitere Fälle von kurialem Interesse an Prophezeiungen - die eine Form pragmatischer Schriftlichkeit darstellten als politische Gutachten zu brennenden Fragen der Zeit mit oft kurzer Verfallszeit - im 13. Jahrhundert vorstellt und eine Typologie von langfristigen und kurzfristigen eschatologischen Vaticinien (je nach Präsenz der Endzeit als solcher) vorschlägt.

Joachim von Fiore, den wichtigsten mittelalterlichen Propheten des lateinisch-christlichen Bereiches, stellt Gian Luca Potestà („Apocalittica e politica in Gioacchino da Fiore") als ungeheuer politischen, mit der Kurie als dem zentralen Machtzentrum eng verbundenen und die Zeitereignisse kommentierenden Denker vor, dessen Schriften teilweise unmittelbar den Wandel der Er- 
eignisse nachzeichnen. Die hohe Bedeutung der Apokalypse in Joachims Geschichtsbild ließ ihn in zahlreichen Schriften Apokalyptik und Politik eng verbinden als ein Theologe, der der Kirche Orientierung bieten wollte gegenüber dem Reich und den Häretikern im Osten (Islam wie Griechen); wobei Joachim grundsätzlich der Gewaltlosigkeit gegenüber der Gewalt den Vorzug gab (außer im Falle des unmittelbar andrängenden Islam).

Felicitas Schmieder („Prophetische Propaganda in der Politik des 14. Jahrhunderts: Johannes von Rupescissa") wendet sich einem Propheten und Kommentator in der Tradition des Joachim von Fiore zu, der in chaotischer ebenso wie hochpolitischer Weise nicht zuletzt den Gegensatz zwischen dem Reich und Frankreich eschatologisch begründete.

Die folgenden drei Beiträge befassen sich mit dem weiten Feld der eschatologischen Vorstellungen im Islam. Wim Raven ( „Ibn Șayyād as an Islamic ,Antichrist'. A Reappraisal of the Texts") legt einleitend zu einer Übersetzung der hadīth-Traditionen (urspr. 8. Jh.) zu Ibn Șayyād als dajjal (dem falschen Messias des Islam) den Forschungsstand dar. Er trägt die wenigen biographischen und handlungsrelevanten Informationen zusammen und nimmt diverse Vorschläge der Forschung zur Lösung des „Rätsels Ibn Șayyād“ auf, so, ob er ein jüdischer dajjal sei oder einer mit dämonischen Zügen, ein Pseudo-Prophet oder Seher - Hinweise, die ihn gefährlich erscheinen ließen und seine Identifikation als dajjal wenigstens suggerierten.

David B. Cook („Apocalyptic Incidents during the Mongol Invasions“) betrachtet die muslimischen Reaktionen auf die mongolische Invasion als genau die Art von Katastrophe, in der apokalyptische und messianische Hoffnungen zu erwarten sind, literarisch wie real. Zwei Schübe - die ersten Einbrüche um 1220 und das weitere Vordringen um 1260 mit der Eroberung Bagdads und dem Ende des abassidischen Kalifats - stehen gegenüber eschatologisch geprägten Widerständen um 1240, der Beobachtung von sich erfüllenden Vorzeichen (himmlischer wie irdischer Feuerzeichen, Endherrscher-Erwartungen) und der interpretatorischen Adaption vor allem der mit Gog und Magog aus dem Alexanderroman verbundenen Türkentradition der hadìth-Traditionen.

Anna A. Akasoy („The muhaqqiq as Mahdi? Ibn Sab'īn and Mahdism Among Andalusian Mystics in the $12^{\text {th }} / 13^{\text {th }}$ Centuries") stellt den westislamischen Sufi Ibn Sab'īn (ca. 1217 - 1270) vor, der zeitgenössisch wie modern als Mystiker mit messianischen Ansprüchen interpretiert wurde, doch sich weder selbst je zum Mahdi erklärte noch in nahestehenden Texten so gesehen wurde. Angesichts der Tatsache, daß er eher als Häretiker erscheint denn als jemand, der sich mit Prophezeiungen beschäftigt, rückt mit Ibn Khaldun die Hauptquelle der Mahdi-Vorwürfe ins Licht: Dessen Interpretation scheint im Kontext der eigenen Befürchtungen und Erwartungen angesichts schiitischen Einflusses und einer eschatologischen Sufi-Bewegung in Andalusien und im Maghreb zustandegekommen zu sein. 
Die letzten vier Beiträge schlagen den chronologischen Bogen in die Frühe Neuzeit. Volker Leppin ( „mit dem künfftigen Jüngsten Tag und Gericht vom sünden schlaff auffgeweckt. Lutherische Apokalyptik zwischen Identitätsvergewisserung und Sozialdisziplinierung, $1548-1618^{\prime \prime}$ ) stellt die lutherische Apokalyptik in zahlreichen Facetten vor. Entsprechend wird Luther als Offenbarer der Institution des Papsttums als Antichrist zu einem Auslöser von Endzeitvorstellungen. Besonders zeigt er ihre sekundäre Funktion als Instrument der Sozialdisziplinierung im eigentlich (im Gegensatz zu den anderen beiden Konfessionen) auf Freiheit beruhenden Luthertum.

Anja Moritz („Endzeit als casus confessionis") analysiert ausführlich die endzeitliche Prägung des Magdeburger lutheranischen Widerstandes gegen das Interim, der zum Casus Confessionis wurde angesichts des Versagens der weltlichen Obrigkeit, die zu Handlangern des Antichrist geworden ist. Vor allem in den Schriften des Nikolaus von Amsdorff, Nikolaus Gallus und Matthias Flacius Illyricus wird der Widerstand als Bastion der Schöpfungsordnung gegen das Chaos des Antichrist gerechtfertigt und zur Notwendigkeit erhoben.

Gerhard Podskalsky SJ („Thomas Malvendas ,De Antichristo“ (Lyon 1647)") stellt Malvendas umfangreiches Sammelwerk literarischer Zeugnisse über den Antichrist vor, mit einem gewissen Fokus auf Schriften patristischer und byzantinischer Provenienz.

Einem ähnlichen Sammelwerk, nun aus lutheranischer Feder, widmete sich schließlich Sabine Schmolinsky („Im Angesicht der Endzeit? Positionen in den Lectiones memorabiles des Johannes Wolff, 1600"). Das riesige Kompendium „prophetischer Materialien aller Art" (ein „Speicher für konfessionspolemisch nutzbare historische Quellen"), das der Lutheraner Johannes Wolff zum Ende des 16. Jahrhunderts in zwei voluminösen Bänden gesammelt hatte, wurde bisher kaum seiner Bedeutung entsprechend gewürdigt. Sie kann einige wichtige Argumentationslinien festhalten, so die Mißstände in der Kirche und auch im Staat (deren Ungleichgewicht an der Konstantinischen Schenkung verdeutlicht wird), die Türkengefahr und den Papst als Antichrist bis hin zu Wolffs eigenen zeit-eschatologischen Deutungen betreffend.

Abschließend bleibt uns die angenehme Pflicht, Frau Iris Heckel und den Herren Daniel Föller und Dr. David Toalster, die bei der Planung und Realisierung der Tagung selbst wie bei der Drucklegung unermüdlich tätig waren, herzlichst zu danken. Nicht minder herzlich ist unser Dank an Frau Dr. Sabine Vogt vom Verlag Walter de Gruyter, deren permanente Unterstützung viel zur erfolgreichen Durchführung der Tagung und dem Druck der Tagungsergebnisse beitrug.

Wolfram Brandes

Felicitas Schmieder 
